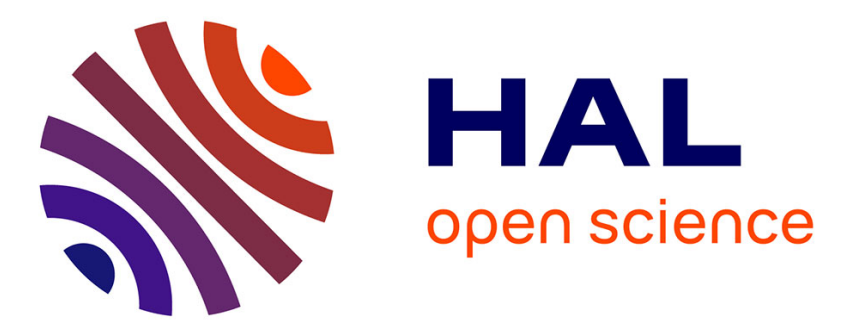

\title{
Transient evolution of the heat transfer and the vapor film thickness at the drop impact in the regime of film boiling
}

Guillaume Castanet, W. Chaze, Ophélie Caballina, R. Collignon, F. Lemoine

\section{- To cite this version:}

Guillaume Castanet, W. Chaze, Ophélie Caballina, R. Collignon, F. Lemoine. Transient evolution of the heat transfer and the vapor film thickness at the drop impact in the regime of film boiling. Physics of Fluids, 2018, 30 (12), pp.122109. 10.1063/1.5059388 . hal-02388404

\section{HAL Id: hal-02388404 https://hal.science/hal-02388404}

Submitted on 2 Apr 2020

HAL is a multi-disciplinary open access archive for the deposit and dissemination of scientific research documents, whether they are published or not. The documents may come from teaching and research institutions in France or abroad, or from public or private research centers.
L'archive ouverte pluridisciplinaire HAL, est destinée au dépôt et à la diffusion de documents scientifiques de niveau recherche, publiés ou non, émanant des établissements d'enseignement et de recherche français ou étrangers, des laboratoires publics ou privés. 


\title{
Transient evolution of the heat transfer and the vapor film thickness at the drop impact in the regime of film boiling
}

\author{
G. Castanet, ${ }^{1, a)}$ W. Chaze ${ }^{2}$ O. Caballina, ${ }^{1}$ R. Collignon, ${ }^{1}$ and F. Lemoine ${ }^{1}$ \\ ${ }^{1}$ Université de Lorraine, CNRS, LEMTA, F-54000 Nancy, France \\ ${ }^{2}$ Division of Combustion Physics, Lund University, P.O. Box 118, 22100 Lund, Sweden
}

(Received 20 September 2018; accepted 3 December 2018; published online 28 December 2018)

\begin{abstract}
When a drop impinges onto a wall heated above the Leidenfrost temperature, a very thin vapor film is formed at the interface between the liquid and the solid substrate. This vapor layer modifies the impact behavior of the drop and induces a significant decrease in heat transfer. A model is proposed for the growth of this vapor layer and its resistance to the heat transfer. The main assumptions are as follows: (i) a uniform but time varying thickness of the vapor film, (ii) a quasi-steady Poiseuille flow inside the vapor film, and (iii) a constant wall temperature. Heat energy and momentum balances are employed to obtain an ordinary differential equation describing the evolution of the vapor film thickness during the drop impact. For droplets injected at a temperature sufficiently lower than the saturation temperature, this equation predicts that the impact velocity has no influence on the thickness of the vapor film. This latter is solely governed by the local heat flux transferred to the liquid, which predominates over the heat flux used for liquid evaporation. An accurate description of the droplet heating is therefore required to complement this model. As an attempt, this description is based upon a one-dimensional analysis, which includes some effects due to the complex fluid flow inside the spreading droplet. Finally, the theoretical model is validated against experiments dealing with millimeter-sized ethanol droplets. Two optical measurement techniques, based on laser-induced fluorescence and infrared thermography, are combined to characterize the heat transfer as well as the thickness of the vapor film. Published by AIP Publishing. https://doi.org/10.1063/1.5059388
\end{abstract}

\section{INTRODUCTION}

Drop impact onto heated surfaces is common in many industrial applications. The ability of sprays to dissipate very high heat fluxes using a limited amount of coolant is extremely acute for spray cooling. ${ }^{1-3}$ In combustion engines, the dispersion of the fuel mist and the enhancement of evaporation resulting from the drop impact are critical to maximize the efficiency of combustion systems and minimize their emissions of pollutants. ${ }^{4}$ In this context, the phenomenon of drop impact has driven the attention of many researchers. However, even though various aspects of the drop impact have been investigated, the understanding of the phenomenon is still limited. For instance, heat transfer coefficients are usually determined experimentally on a global scale, based on the mean parameters of the spray (for example, the mean size and velocity of the incident droplets, or the coolant volumetric flux). Such correlations can serve well for an intended configuration of spray, but in general they lack generality to adapt to other spray configurations. There is therefore an important need to obtain a glimpse of the underlying physics by carrying out investigations at the level of individual droplets.

Numerous studies have focused on the drop impact onto an isothermal surface, which does not require consideration of heat transfer and phase change. In these studies, the

a)Electronic mail: guillaume.castanet@univ-lorraine.fr emphasis was mainly placed on the drop behavior at the impact by characterizing the diameter and the velocity of the outcoming droplets (partial/total rebound and splashing $)^{5}$ and the spreading of the liquid film. ${ }^{6}$ These investigations mainly aimed at determining the influence of the dynamical parameters (velocity and diameter), ${ }^{5}$ and the physical properties of the liquid (viscosity and surface tension) ${ }^{5}$ and of the solid surface (surface roughness and wettability) $)^{5,7,8}$ on the impact process. These studies must be re-examined when the wall is heated above the boiling point of the liquid. Even when the temperature of the solid substrate exceeds the saturation temperature of the liquid by a few degrees, boiling considerably changes the heat transfer and the dynamics of the drop. As for pool boiling, the boiling curve (heat flux versus wall superheat) is a complex non-monotonic function of the wall temperature (see Fig. 1 in Ref. 9, p. 105). It can be categorized into boiling regimes: (i) natural convection boiling, (ii) nucleate boiling, (iii) transition boiling, and (iv) film boiling. In the regime of film boiling, the liquid does not remain in contact with the solid. A vapor layer is formed underneath the droplet (Leidenfrost effect) and prevents at any time a contact between the liquid and the solid surface. ${ }^{10}$ Therefore, only two dynamical behaviors are observed: rebound (with or without partial fragmentation) and splashing. Various aspects of the Leidenfrost phenomenon have been investigated, for example, the droplet spreading over the vapor film, ${ }^{11-13}$ the transition between rebound and splashing regimes, ${ }^{14}$ the contact time of the drop near the wall, ${ }^{15,16}$ the spreading time, ${ }^{11,15,17}$ the restitution of 
velocity in the case of a rebound, ${ }^{13,16}$ the characteristics of secondary droplets in a splashing, ${ }^{14}$ and the dynamic Leidenfrost temperature. ${ }^{18,19}$ When the Leidenfrost phenomenon is concerned, it should be mentioned that it does not exclusively concern drops impinging onto a hot solid surface. It can manifest under many different situations. While the Leidenfrost effect limits the drop cooling of hot surfaces, it can be advantageous for other applications. Janssens et al. ${ }^{20}$ reported the formation of a vapor film at the interface between a liquid drop and a much hotter liquid surface. The Leidenfrost effect prevents the coalescence of the liquids and even self-propulsion of the drop can occur. Berry et al. ${ }^{21}$ took advantage of film boiling at the surface of a hot rigid sphere immersed into a liquid to reduce its drag coefficient. Sobac et al. ${ }^{22}$ described the self-propulsion of Leidenfrost drops placed onto a solid substrate with a temperature gradient of the surface. In the work of Dupeux et al., ${ }^{23}$ the propulsion of Leidenfrost drops is obtained by modifying the topology of the solid surface. An "inverted" Leidenfrost effect is described by Antonini et $a l .,{ }^{24}$ when a dry ice substrate sublimes due to the presence of a drop on the top of it. The release of carbon dioxide gas is able to induce the bouncing of a drop that impacts the dry ice surface.

The main subject of the present study is the introduction of a predictive theoretical model for the heat transfer during a drop impact onto a flat solid surface in the film boiling regime. The presence of a vapor film is known to drastically reduce the heat transfer. It is therefore very important to have a model for the thickness of the vapor film. Some theoretical models are available in the literature for sessile droplets, deposited without velocity on a solid surface. Biance et al $^{25}$ derived some scaling laws for the vapor film thickness. From experiments, they evidenced two distinctive evolutions of the vapor thickness with the drop size, depending on the drop shape: a puddle or a quasi-spherical drop. Sobac et al. ${ }^{26}$ proposed a model based upon lubrication theory which accounts for the curvature of the liquid/vapor interface. This provides good agreement with experimental studies showing the presence of a vapor pocket underneath the droplet and a neck in the profile of the vapor film. Sobac et al. ${ }^{22}$ recently extended this approach to the case of self-propelled Leidenfrost drops. However, the adaptation of these theoretical descriptions to the case of a drop impact is not straightforward, since the force acting upon the vapor film and the spreading evolution of the droplet are considerably changed. Furthermore, the transient heating of the drop must also be taken into account in the case of an impact. Breitenbach et $a l .{ }^{27}$ derived a theoretical model for the heat transfer and the growth rate of the vapor film in the case of a drop impact. According to this model, the evolution of the vapor film thickness can be described in a similar way as the growth of a vapor bubble in boiling liquid. Indeed, Breitenbach et al. ${ }^{27}$ postulated that the growth rate of the vapor film is governed by the rate of liquid vaporization, and they evaluated the heat flux devoted to vaporization by $q_{v}=\rho_{l} L v \mathrm{~d} h / \mathrm{d} t$, where $h$ is the thickness of the vapor film, $\rho_{l}$ is the liquid density, and $L v$ is the latent heat of vaporization. Their model of heat transfer was compared to the heat flow measured globally at the impact of a polydisperse spray, but this remains a rather indirect method of validation. In the case of a spray, additional assumptions must be made concerning the interaction between the impacts of neighbor droplets, the cooling being less effective when impacts are separated by a short distance and when they occur within a short interval of time.

Data collected at the scale of a single drop provide an unparalleled source of information for assessing fundamental models of drop impact. In this study focused on heat and mass transfers in the film boiling regime, it is very important to characterize the rate of heating of the drop, the cooling of the solid surface, and the thickness of the vapor film. To this end, two measurement techniques were developed specifically in this study. A first technique based on two-color laser-induced fluorescence (2cLIF) imaging allows us to determine the temperature of the liquid drop during the impact process. ${ }^{28}$ In parallel, infrared (IR) thermography is used to characterize the temperature of the solid surface. Then, an inverse heat conduction problem (IHCP) is solved to estimate the heat flux from the solid wall removed by the droplet and the thickness of the vapor film. ${ }^{29}$

\section{EXPERIMENTAL CHARACTERIZATION OF THE HEAT TRANSFERS AND THE VAPOR FILM THICKNESS}

\section{A. Experimental setup and droplet generation}

A $400 \mu \mathrm{m}$ base diameter needle attached to a syringe is used to produce ethanol drops with a diameter $d_{0}=1.95 \mathrm{~mm}$ (Fig. 1). The drop hanging on the end of the needle detaches itself when its weight equals the surface tension force. The frequency of the droplet detachment is controlled by adjusting the liquid flow rate with the syringe driver. Free-falling droplets impinge a sapphire window $(2.54 \mathrm{~cm}$ in diameter and $5 \mathrm{~mm}$ thick) placed on a steel holder which is heated using cartridge heaters. Owing to the large thermal conductivity of

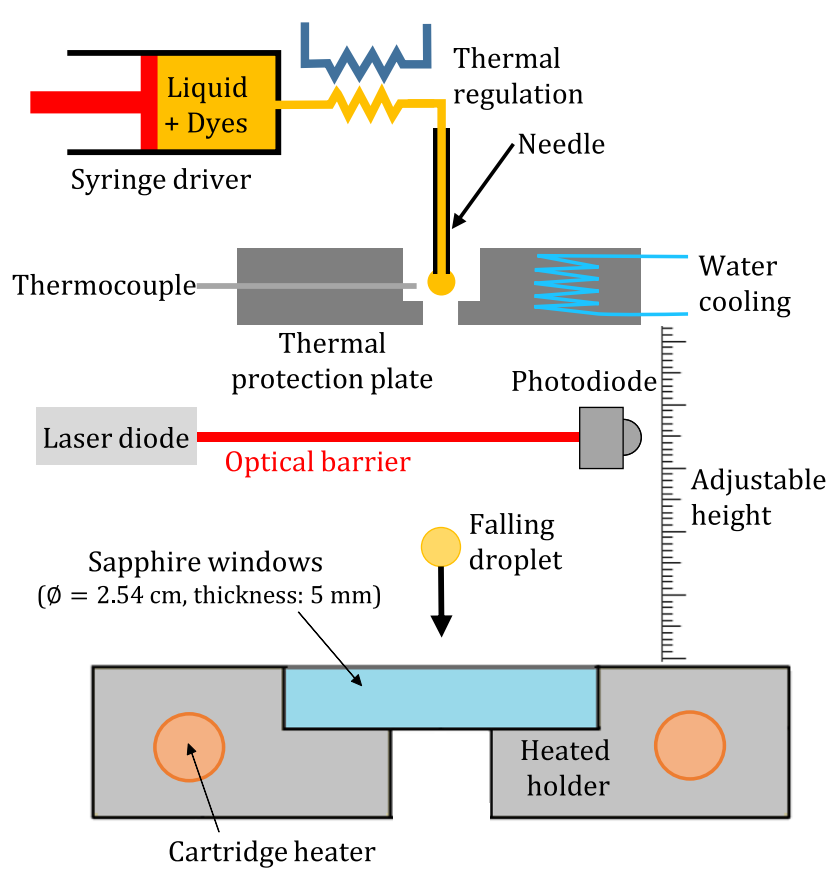

FIG. 1. The experimental setup. 
sapphire [about $40 \mathrm{~W} /(\mathrm{mK})$ at $20^{\circ} \mathrm{C}$ ], the temperature of the solid surface is almost uniform and equal to that of the steel holder in the absence of droplets. A thermal protection is necessary to prevent the liquid inside the needle from being heated by the hot air plume rising from the sapphire window. The tip of the needle is inserted into a cavity of a few millimeters arranged inside a metallic plate which is cooled down by water circulation. Moreover, the liquid passes into a heat exchanger before entering the needle. The needle and the protection plate are moved up and down to modify the impact velocity $V_{d 0}$ and thus adjust the value of the Weber number $\left(W e=\rho_{l} V_{d 0}^{2} d_{0} / \gamma\right.$, with $\gamma$ denoting the surface tension of the liquid), which is commonly used to describe the dynamical behavior of a spreading drop. An aperture managed in the steel holder allows having an optical access from below for bottom-view visualization and backside illumination of the spreading droplet for side-view visualization.

\section{B. Measurement of the droplet temperature}

Only a brief overview of the technique used for measuring the temperature of the droplet is recalled in this section. Details of the method can be found in Ref. 28. The two-color laserinduced fluorescence ( $2 \mathrm{cLIF}$ ) thermometry has already been used in previous studies to determine the heating of droplets while they are impinging onto a hot solid substrate in the film boiling regime. The first measurements were limited to the volume-averaged temperature of the droplets. ${ }^{30,31}$ Significant improvements were made recently by Chaze et ll $^{28}$ in order to obtain temporally and spatially resolved images of the temperature field within the spreading droplet.

Two fluorescent dyes, namely, sulforhodamine 640 (SR640) and disodium fluorescein (FL), are dissolved into the liquid (presently ethanol). When illuminated by a pulsed Nd:YAG laser at $532 \mathrm{~nm}$, FL emits a fluorescence signal which

a)

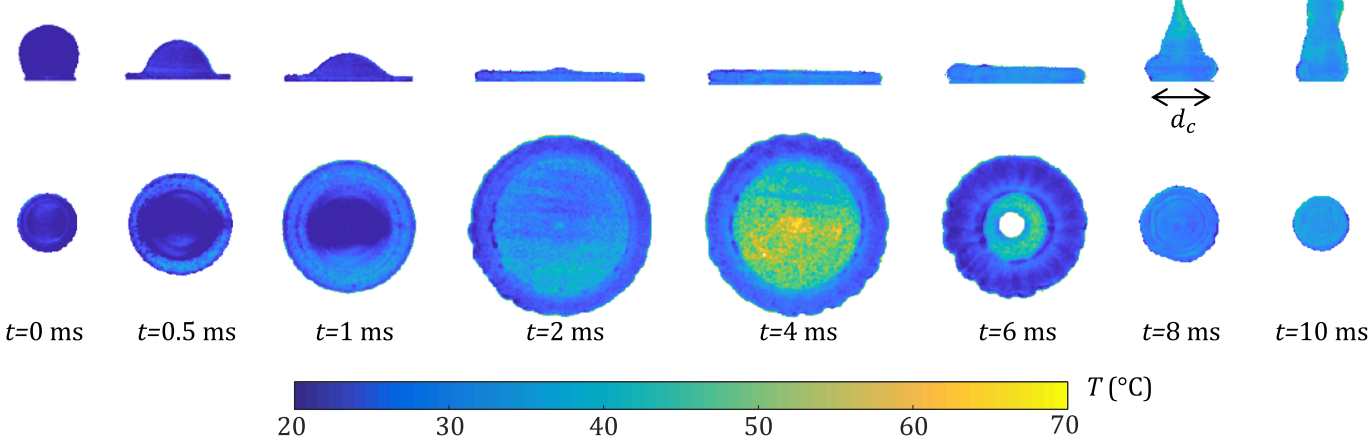

b)

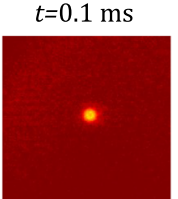

$t=4.1 \mathrm{~ms}$

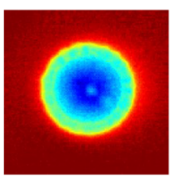

c)
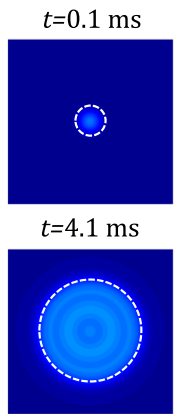

$t=0.9 \mathrm{~ms}$

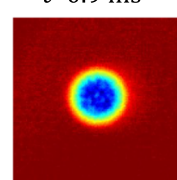

$t=4.9 \mathrm{~ms}$

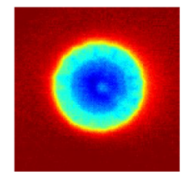

$t=0.9 \mathrm{~ms}$

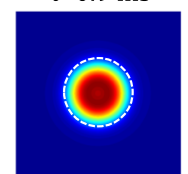

$t=4.9 \mathrm{~ms}$

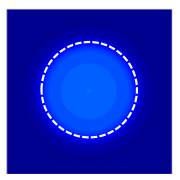

$t=1.7 \mathrm{~ms}$

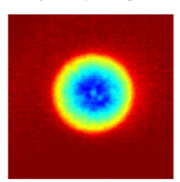

$t=6.5 \mathrm{~ms}$

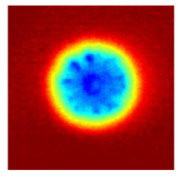

$t=1.7 \mathrm{~ms}$

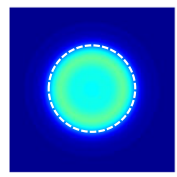

$t=6.5 \mathrm{~ms}$

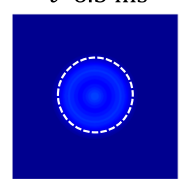

$t=2.5 \mathrm{~ms}$

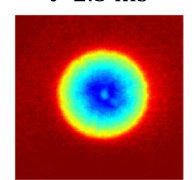

$t=8.1 \mathrm{~ms}$

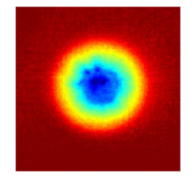

$t=2.5 \mathrm{~ms}$

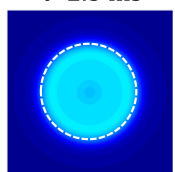

$t=8.1 \mathrm{~ms}$

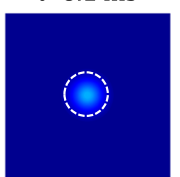

$t=3.3 \mathrm{~ms}$

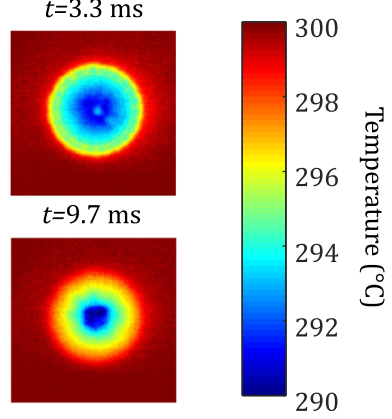

$t=3.3 \mathrm{~ms}$

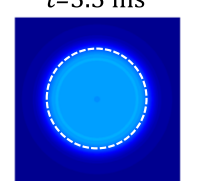

$t=9.7 \mathrm{~ms}$
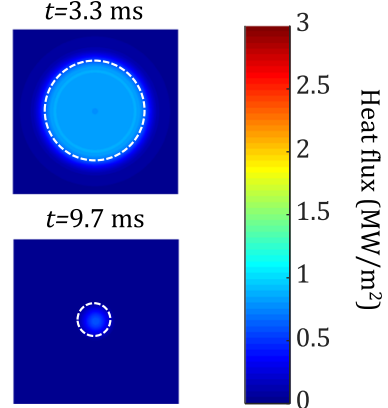

FIG. 2. Characterization of heat transfer in the case of an ethanol droplet impinging onto a sapphire window at $W e=90\left(T_{d 0}=20{ }^{\circ} \mathrm{C}, T_{w}=300{ }^{\circ} \mathrm{C}\right.$, and $d_{0}$ $=1.95 \mathrm{~mm}$ ). (a) Temperature distribution in the liquid drop obtained by $2 \mathrm{cLIF}$ imaging. The diameter of the apparent "contact" line $d_{c}$ can be deduced from the sideview images. (b) Temperature distribution at the solid surface measured by infrared thermography. (c) Heat flux from the solid surface reconstructed by solving the IHCP. The region of most efficient heat exchange $S_{e}$ is indicated by the dotted lines. 
strongly increased with the temperature. However, the intensity of the FL signal is affected by light scattering at the droplet surface which is deforming during the impact. A second dye, namely, SR640, is used as a reference for measuring the temperature. The signal of SR640 is almost insensitive to the temperature and is red-shifted of a few tens of nm compared to FL, which allows recording images of FL and SR640 emissions in separate spectral bands using two cameras equipped with appropriate bandpass filters. Doing the ratio of the images allows eliminating the dependence on the droplet shape, since the effect of light scattering is almost identical for the two detection bands. The ratio is converted into temperature by means of a calibration performed prior to the experiments in a glass cuvette where the liquid is regulated in temperature. As illustrated in Fig. 1, an optical barrier consisting of a laser diode and a photodiode makes it possible to detect the passage of the falling drop with an accuracy of $10 \mu$ s to trigger the image acquisition. The mass fraction of the fluorescent dyes in the liquid (FL and SR640 combined) is equal to $2.2 \times 10^{-5}$. This very low value ensures that the dyes do not modify the drop behavior and the heat and mass transfers. No change in the drop spreading and in the cooling of the solid surface was noticed with the addition of FL and SR640. Given the relatively large molar mass of FL and SR640, the dyes remain inside the liquid phase during the evaporation of the drop.

An example of temperature maps is presented in Fig. 2(a). It corresponds to the case of an ethanol drop impinging onto the sapphire window at $T_{w}=300{ }^{\circ} \mathrm{C}$ and a value of the Weber number We equal to 90 . A short time after the beginning of the impact, the droplet takes the shape of a thin lamella of liquid surrounded by an annular rim. In the first times, heating seems to proceed at a faster rate in the bounding rim, where hot liquid accumulates and mixes with colder liquid already there. As time goes by, the liquid lamella becomes thinner and thinner and the temperature in its inside increases very sharply. The temperature of the lamella gets close to the boiling point of ethanol $\left(T_{\text {Sat }}=78^{\circ} \mathrm{C}\right)$ at $t=4 \mathrm{~ms}$. Nonetheless, the lamella does not account for a significant part of the drop volume at the end of the spreading. The receding of the drop edge accelerates the internal mixing of the liquid. At $t=10 \mathrm{~ms}$, the drop leaves the solid surface with a rather uniform temperature, even though the top of the vertically elongated droplet has a slightly higher temperature.

\section{Heat flux reconstruction at the solid surface}

The temperature of the impact surface $T_{w, s}$ is characterized by means of an IR camera. The acquisition by the IR camera is triggered by the passage of the droplet across the optical barrier. Given that sapphire is transparent in the IR domain up to $7 \mu \mathrm{m}$, the coating of the window is required to perform these measurements. The upper face of the sapphire window, where impacts take place, is coated with a nanolayer of TiAlN (300 nm thickness) which is resistant to oxidation and thermal stress at the drop impact. Furthermore, this material has a high emissivity in the detection band of the camera ( $\varepsilon \approx 0.93$ ). As a benefit of a high emissivity, the integration time of the camera can be shortened to a few tens of $\mu \mathrm{s}$. Moreover, reflections of environmental radiations (especially those coming from the heated holder) have a very limited contribution to the signal and can be ignored in the processing of the images. Figure 2(b) shows a typical set of temperature images recorded with the IR camera. In this example of an impinging ethanol droplet, cooling of the sapphire surface does not exceed $10^{\circ} \mathrm{C}$. To obtain this sequence, the acquisition rate of the camera was fixed at $1250 \mathrm{fps}$, but this was at the expense of the image resolution which is limited to $160 \times 128$ pixels.

In order to reconstruct the heat flux distribution at the solid surface, an inverse heat conduction problem (IHCP) is solved. The transient heat conduction within the sapphire substrate is taken into account using the so-called quadrupole method. ${ }^{29}$ This approach provides an analytical relationship between the temperature $T_{w, s}$ and the heat flux $q_{w}$ at the front surface of the solid wall, where the drop impact takes place. To limit the noise inherent to the inversion, the problem is considered axisymmetric. This approximation appears to be well justified in Fig. 2(b) where the temperature distribution on the solid surface seems rather axisymmetric. Pixels situated at the same distance from the center of the impact are averaged before solving the IHCP. A maximum of heat flux is reached very rapidly after the beginning of the impact [Fig. 2(c)] and then the heat flux decreases progressively with time.

\section{Evaluation of the vapor film thickness}

Given the small thickness of the vapor film, thermal conduction is predominant in the direction perpendicular to the solid surface. ${ }^{22,25-27,32}$ As a result, the temperature in the vapor film varies linearly between the liquid surface at the saturation temperature $T_{S a t}$ and the solid surface at $T_{w, s}$. Knowing the value of the heat flux $q_{w}$ from the resolution of the IHCP, the thickness of the vapor film $h$ can be determined by

$$
h=\lambda_{v} \cdot \Delta T / q_{w},
$$

where $\lambda_{v}$ is the thermal conductivity of the vapor and $\Delta T=T_{w, s}$ $-T_{\text {Sat }}$. Figure 3 shows profiles of the vapor film at different times corresponding to the same drop impact as in Fig. 2. It can be observed that the vapor film is steadily growing with time until $t \approx 5 \mathrm{~ms}$ and then the thickness increases very rapidly

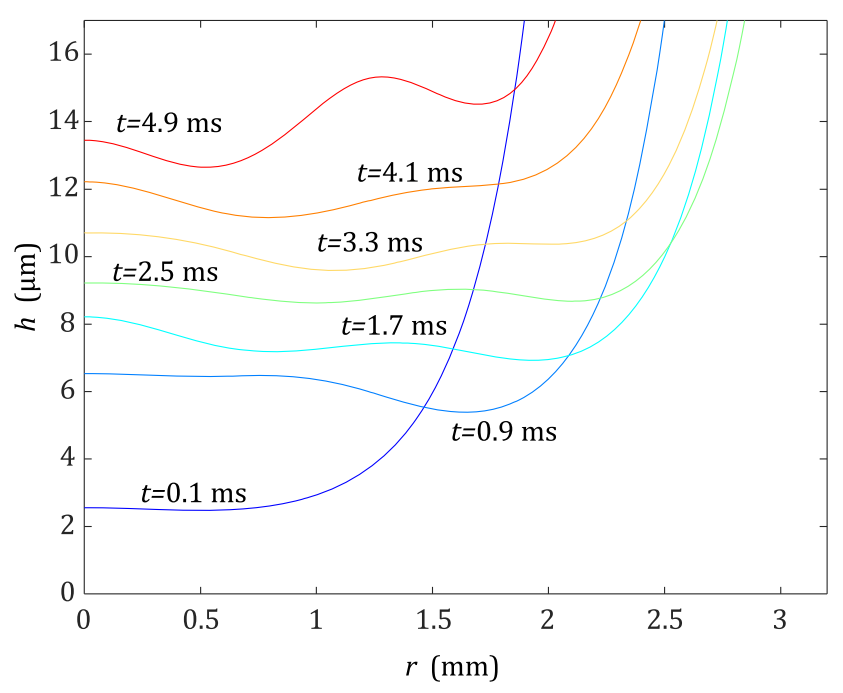

FIG. 3. Evolution of the vapor film thickness $h$ for $W e=90$ in the case of an ethanol droplet $\left(T_{w}=300^{\circ} \mathrm{C}, T_{d 0}=20^{\circ} \mathrm{C}\right.$, and $\left.d_{0}=1.95 \mathrm{~mm}\right)$. 
because of the formation of a hole in the middle of the liquid lamella [Fig. 2(a)]. At $t=0.9 \mathrm{~ms}$, the vapor film profile takes a characteristic shape with a bottleneck at the edge. Globally, the difference in thickness between the center and the edge of the film remains very moderate, which justifies the assumption of a uniform thickness of the vapor film made in Sec. III.

\section{MODELING OF THE VAPOR FILM THICKNESS}

The configuration considered for the modeling is presented in Fig. 4. The problem is considered to be axisymmetric, and the vapor film thickness $h$ is supposed to be uniform but time-dependent. The area of effective heat transfer between the droplet and the solid surface is equal to $\pi d_{c}^{2} / 4$, where $d_{c}$ is sometimes called "contact" diameter. The spreading diameter $d_{x}$ is usually close but not exactly equal to $d_{c}$, the difference being due to the rounded edge of the spreading droplet.

\section{A. Flow motion inside the vapor layer}

Considering that $d_{c}$ is, at any time, much larger than $h$, the dynamics of the vapor in the film can be described within the scope of the lubrication theory. ${ }^{22,26,32}$ Hence, the derivatives of the velocity with respect to $r$ are small in front of the derivatives with respect to $z$, and the inertial term $(\mathbf{u} \cdot \nabla \mathbf{u})$ can be neglected in the Navier-Stokes equations. Taking into account the axisymmetry of the spreading drop, the momentum balance in the radial direction $r$ can be expressed as

$$
\frac{\partial v_{r}}{\partial t}=-\frac{1}{\rho_{v}} \frac{\partial p}{\partial r}+v_{v} \frac{\partial^{2} v_{r}}{\partial z^{2}},
$$

where $v_{r}$ is the radial component of the velocity inside the vapor film. $\rho_{v}$ and $v_{v}$ are the density and the kinematic viscosity of the vapor, respectively. In this expression, the unsteady term $\partial v_{r} / \partial t$ is negligible in comparison to the viscous term. A rough estimate can be made assuming that $\partial v_{r} / \partial t \sim v_{r} / t_{i}$ and $v_{v}$. $\partial^{2} v_{r} / \partial z^{2} \sim v_{v} \cdot v_{r} / h^{2}$, where the characteristic time of an impact $t_{i}$ is a few ms, $h \sim 1 \mu \mathrm{m}$, and $v_{v} \approx 10^{-4} \mathrm{~m}^{2} / \mathrm{s}$. This leads to a viscous term typically $10^{4}$ times larger than the unsteady one. The velocity field is thus a Poiseuille flow, which is the solution of the equation

$$
\frac{1}{\rho_{v}} \frac{\partial p}{\partial r}=v_{v} \frac{\partial^{2} v_{r}}{\partial z^{2}}
$$

Although the thickness of the film $h$ varies with time, the problem can be treated as a quasi-steady one. The mass conservation applied to the vapor film yields

$$
\pi r^{2} \dot{m}^{\prime \prime}=2 \pi r h \rho_{v} \bar{v}_{r}(r)
$$

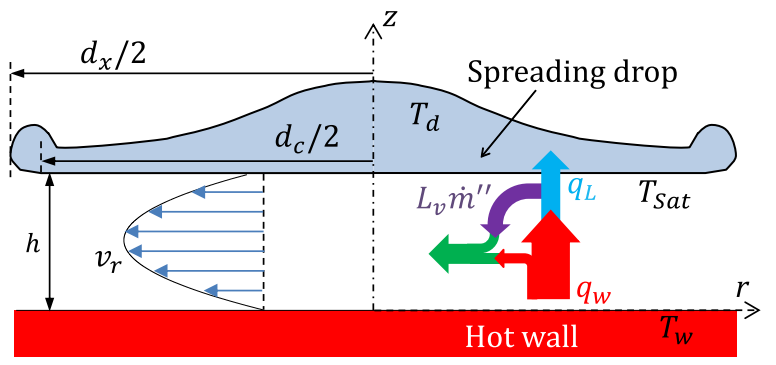

FIG. 4. Schema of the configuration considered for the modeling of the vapor film. where $\bar{v}_{r}(r)=\frac{1}{h} \int_{0}^{h} v_{r} d z$ is the mean radial velocity of the vapor in the film and $\dot{m}^{\prime \prime}$ is the mass flow rate per unit area of the vapor released underneath the droplet. According to Eq. (4), the radial velocity $v_{r}$ increases linearly with $r$. To satisfy Eqs. (3) and (4), the radial velocity $v_{r}$ must be in the form

$$
v_{r}=\left(b z+c z^{2}\right) \cdot r .
$$

Applying the continuity equation, the component of the velocity perpendicular to the wall can be obtained as

$$
v_{z}=-\int \frac{1}{r} \frac{\partial r v_{r}}{\partial r} d z=-b z^{2}-\frac{2}{3} c z^{3},
$$

where $b$ and $c$ are determined in regard to the boundary conditions. When the droplet spreads, a thin liquid lamella bounded by a rim is formed. The flow within this lamella can be approximated by the asymptotic flow solution proposed by Yarin and Weiss $^{33}$

$$
v_{r, l}=\frac{r}{t}, v_{z, l}=\frac{-2 \xi}{t},
$$

where $\xi=z-h$. The subscript $l$ refers to the liquid phase. Equation (7) corresponds to a non-viscous flow solution. The slip velocity of the liquid/vapor interface is commonly assumed to be not affected by the vapor flow. The liquid drop moves almost freely over the vapor film. Numerous studies have emphasized a significant reduction of friction associated with the presence of a vapor film. ${ }^{21}$ In the case of a drop impact, the vapor film is squeezed and the radial velocity $v_{r}$ of the vapor is usually much larger than the liquid velocity $v_{r, l}$. Therefore, it has been suspected that the liquid can be entrained radially by the vapor flow. In this scenario, according to Castanet et al., ${ }^{11}$ the radial velocity at $\xi=0$ can be determined by

$$
v_{r, l}=\alpha \frac{r}{t} \text { at } \xi=0,
$$

where $\alpha$ is a parameter that can be slightly larger than 1 . The following conditions are considered at the liquid/vapor interface:

$$
\begin{gathered}
v_{r}=v_{r, l} \text { at } z=h, \\
\rho_{v, s} \cdot\left(v_{z}-\frac{\mathrm{d} h}{\mathrm{~d} t}\right)=-\dot{m}^{\prime \prime} \text { at } z=h .
\end{gathered}
$$

In this expression, $\rho_{v, s}$ is the vapor density at the liquid/vapor interface where $T=T_{\text {Sat }}$. Using Eqs. (5)-(10), the parameters $b$ and $c$ can be determined by

$$
\begin{gathered}
c=\frac{\alpha}{h^{2} t}-\frac{b}{h}, \\
b=-\frac{3}{h^{2}}\left(\frac{\mathrm{d} h}{\mathrm{~d} t}-\frac{\dot{m}^{\prime \prime}}{\rho_{v, s}}+\frac{2}{3} \frac{\alpha h}{t}\right) .
\end{gathered}
$$

\section{B. Energy balance applied to the vapor film}

The heat flux taken from the wall $q_{w}$ is used first to heat up the liquid in the drop, to vaporize the liquid, and finally to increase the temperature of the released vapor up to the average temperature in the vapor film which is denoted $T_{\text {film }}$. Hence, the balance of energy flux can be summarized as follows:

$$
\begin{gathered}
q_{w}=q_{L}+L v_{e} \cdot \dot{m}^{\prime \prime}, \\
L v_{e}=L v+\left(C p_{v} \cdot T_{f i l m}-C p_{v, s} \cdot T_{\text {Sat }}\right) .
\end{gathered}
$$


In Eq. (13), $q_{L}$ corresponds to the heat flux entering into the liquid drop. This heat flux is responsible for the temperature increase in the droplet during the impact. Since the variation in temperature in the vapor film is linear along the $z$-axis, the average temperature of the vapor $T_{\text {film }}$ can be well approximated by $T_{f i l m}=\left(T_{S a t}+T_{w, s}\right) / 2 . C p_{v, s}$ and $C p_{v}$ denote the heat capacity of the vapor at $T_{S a t}$ and $T_{\text {film }}$, respectively. Using Eqs. (1) and (13), the vapor mass flowrate $\dot{m}^{\prime \prime}$ can be determined by

$$
L v_{e} \cdot \dot{m}^{\prime \prime}=\frac{\lambda_{v} \Delta T}{h}-q_{L}
$$

\section{Momentum balance applied to the vapor film}

The reaction force $F$ of the vapor film on the impinging droplet is due to the pressure inside the vapor film. Using Eq. (5), the integration of Eq. (3) allows obtaining the following expression of the pressure:

$$
p(r)-p(r=0)=\mu_{v} c r^{2} .
$$

On the outer edge of the vapor film at $r=d_{c} / 2$, the pressure returns to the external pressure, meaning that $p=0$. This yields

$$
p(r)=\mu_{v} c \cdot\left(r^{2}-\frac{d_{c}^{2}}{4}\right) .
$$

The reaction force $F$ can be determined by

$$
F=2 \pi \int_{r=0}^{d_{c} / 2} p(r) r d r=-\frac{\pi}{32} \mu_{v} c d_{c}^{4} .
$$

Using Eqs. (11) and (12), the following expression of the force $F$ can be obtained:

$$
F=\frac{3 \pi}{32} \mu_{v} \frac{d_{c}^{4}}{h^{3}}\left(\frac{\dot{m}^{\prime \prime}}{\rho_{v, s}}-\frac{\alpha h}{t}-\frac{\mathrm{d} h}{\mathrm{~d} t}\right) .
$$

This expression of $F$ is equivalent to the Stefan-Reynolds force opposed to the approach of two parallel disks ${ }^{34}$ when neglecting the liquid vaporization $\dot{m}^{\prime \prime}$ and the sliding of the liquid/vapor interface (i.e., $\alpha=0$ and $\dot{m}^{\prime \prime}=0$ ). Based on Eqs. (15) and (19), the evolution of the vapor film thickness can be determined by solving

$$
\frac{\mathrm{d} h}{\mathrm{~d} t}=-\frac{\alpha h}{t}+\frac{1}{\rho_{v, s} L v_{e}} \cdot\left(\frac{\lambda_{v} \Delta T}{h}-q_{L}\right)-\frac{32}{3 \pi} \frac{h^{3} F}{\mu_{v} d_{c}^{4}} .
$$

It is easy to verify that the case of the sessile drop can be recovered from Eq. (20). To mimic a sessile drop, we must set that $F=m \cdot g$ with $m$ being the mass of the drop, $q_{L}=0$, and $\mathrm{d} h / \mathrm{d} t=0$. It is also assumed that $\alpha=0$ (negligible small velocity at the liquid surface). Under these conditions, Eq. (20) becomes

$$
m \cdot g=\frac{3 \pi}{32} \mu_{v} \frac{d_{c}^{4}}{h^{4}} \frac{\lambda_{v} \Delta T}{\rho_{v, s} L v_{e}} .
$$

The drop takes a flattened shape, ${ }^{25}$ when the drop size is much larger than the capillary length defined by $l_{c}=\sqrt{\gamma / \rho_{l} g}$, where $\gamma$ is the surface tension of the liquid. Its height is roughly equal to $2 l_{c}$ and its mass can be evaluated by $m=\frac{\pi}{2} \rho_{l} d_{c}^{2} l_{c}$. Using Eq. (21), the thickness of the vapor film can be determined by

$$
h=\left(\frac{3}{4} \frac{\mu_{v} \lambda_{v} \Delta T}{L v_{e} \rho_{l} \rho_{v, s} g l_{c}}\right)^{1 / 4} \cdot\left(\frac{d_{c}}{2}\right)^{1 / 2} .
$$

This expression is identical to that derived by Biance et al. ${ }^{25}$ for the case of the sessile drop.

In the general case, solving Eq. (20) requires an evaluation of $q_{L}, d_{c}$, and $F$. The modeling of the heat flux entering into the droplet $q_{L}$ is the subject of Sec. IV.

\section{MODELING OF THE DROP HEATING}

A one-dimensional model for the droplet heating can be associated with the previous description of the vapor film. ${ }^{27}$ The droplet is assimilated to a semi-infinite liquid medium (Fig. 5). The thickness of the thermal boundary layer, which develops inside the drop at the interface with the vapor film, is small in comparison to the radial dimension of the spreading drop. Therefore, the thermal gradient in the $z$ direction $\partial T / \partial z$ is dominant relative to that in the radial direction $\partial T / \partial r$. The heat equation can be written as

$$
\frac{\partial T}{\partial t}-v_{z, l}(\xi) \cdot \frac{\partial T}{\partial \xi}=a_{l} \frac{\partial^{2} T}{\partial \xi^{2}},
$$

where $\xi=z-h, a_{l}$ is the thermal diffusivity of the liquid, and $v_{z, l}$ is the $z$ component of the liquid velocity, which can be evaluated using Eq. (7). Hence, the convection induced by the liquid flow within the lamella is taken into account in Eq. (23). At the liquid/vapor interface, the temperature is imposed by the liquid-vapor saturation

$$
T=T_{\text {Sat }} \text { at } \xi=0 .
$$

Assuming that the thickness of the thermal layer is small compared to the drop thickness, one can expect

$$
T=T_{d 0} \text { at } \xi \rightarrow \infty,
$$

where $T_{d 0}$ is the drop temperature before impinging onto the hot solid surface. As presented by Breitenbach et al., ${ }^{27}$ the above problem can be solved analytically. The temperature field inside the spreading drop is determined by

$$
T(\xi, t)=T_{\text {Sat }}+\left(T_{d 0}-T_{\text {Sat }}\right) \cdot \operatorname{erf}\left(\frac{\sqrt{5} \xi}{2 \sqrt{a_{l} t}}\right) .
$$

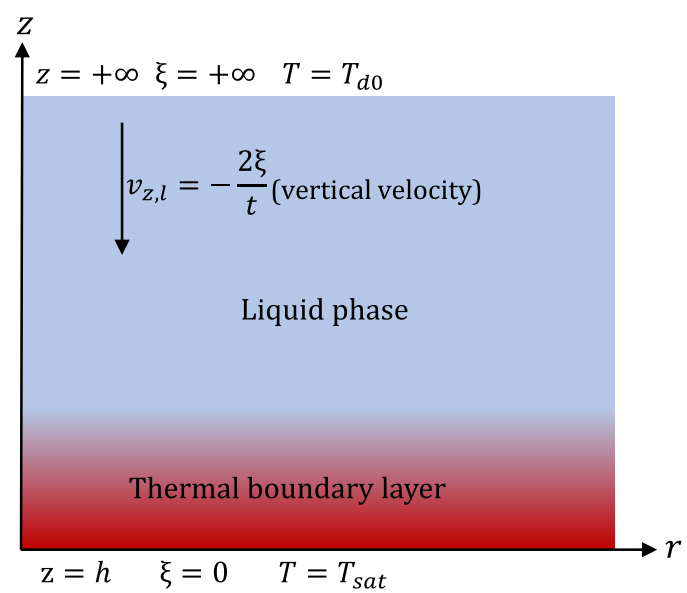

FIG. 5. View of the configuration considered for the modeling of the droplet heating. 


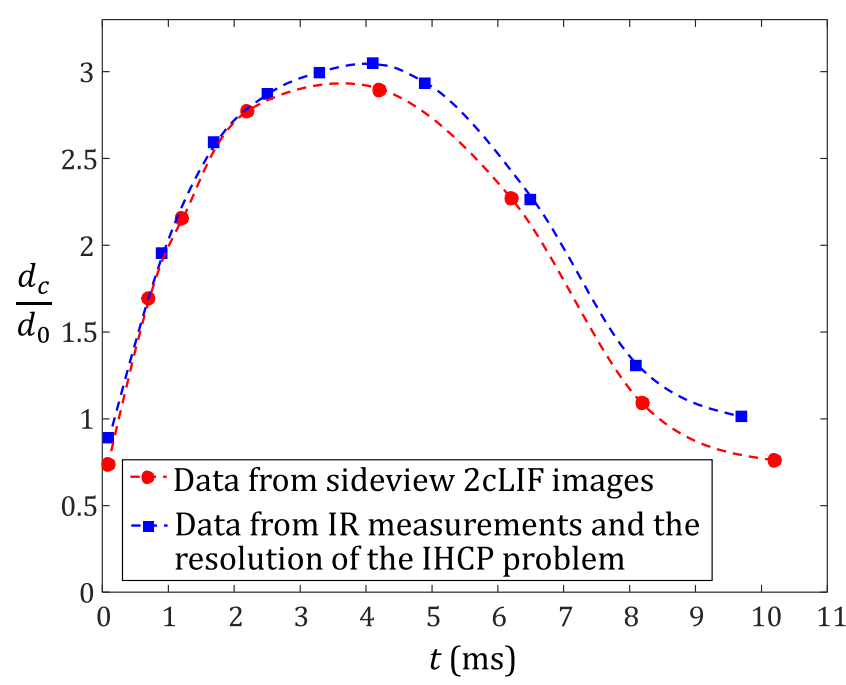

FIG. 6. Time evolution of the diameter $d_{c}$ of the region of most efficient heat exchange for the same example as in Fig. 2. (Red) data obtained from sideview 2cLIF images [Fig. 2(a)]; (blue) data from the distribution profiles of $q_{w}$ reconstructed by solving the IHCP [Fig. 2(c)].

The heat flux entering the liquid phase can be calculated from the following expression:

$$
\begin{gathered}
q_{L}=\left.\lambda_{l} \frac{\mathrm{d} T}{\mathrm{~d} \xi}\right|_{\xi=0^{+}}=C_{q_{L}} t^{-1 / 2}, \\
C_{q_{L}}=\frac{\sqrt{5} e_{l}\left(T_{S a t}-T_{d 0}\right)}{\sqrt{\pi}} .
\end{gathered}
$$

Here $e_{l}=\sqrt{\rho_{l} C p_{l} \lambda_{l}}$ is the thermal effusivity of the liquid. In the frame of this one-dimensional description of drop heating, the increase $\Delta T_{d}$ in the mean temperature of the drop $T_{m}$ can be evaluated by

$$
\Delta T_{d}=T_{m}-T_{d 0}=\frac{1}{m C p_{l}} \int_{0}^{t} q_{L}(t) \cdot S_{e}(t) \mathrm{d} t,
$$

where $S_{e}$ is the effective surface of heat exchange between the droplet and the solid substrate, i.e., $S_{e}=\pi d_{c}^{2} / 4$. The time evolution of $d_{c}$ can be evaluated using the theoretical approach developed by Castanet et al., ${ }^{11}$ which is based on

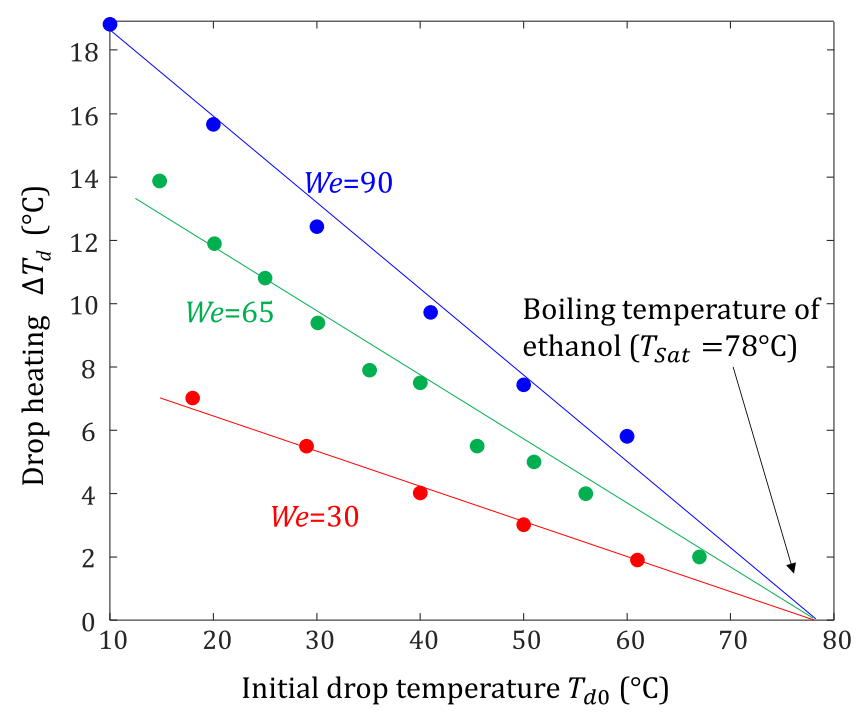

FIG. 8. Experimental data showing the influence of the initial temperature $T_{d 0}$ on drop heating $\Delta T_{d}$.

the application of momentum conservation to the rim bounding the liquid lamella. In the following, $d_{c}$ is evaluated from the sideview images taken by laser-induced fluorescence, which allows evaluating the length of the "apparent contact" line between the solid surface and the drop [Fig. 2(a)]. On the one hand, it is also possible to estimate the area $S_{e}$ of most efficient heat transfers directly from the reconstructed distributions of the wall heat flux $q_{w}$ [Fig. 2(c)] and then to determine its diameter. Figure 6 shows that these two approaches give roughly equivalent results. As $q_{w}$ decreases rapidly with time, the capture of the contour of $S_{e}$ becomes less and less accurate. This certainly explains the slightly larger diameter obtained using the reconstructed images of $q_{w}$ [Fig. 2(c)].

The above-described model of drop heating can be validated against the average temperature in the maps obtained experimentally by laser-induced fluorescence [Fig. 2(a)]. The results of this comparison are presented in Fig. 7 in the case of three different values of the Weber number We. A good agreement between the experimental data and the results of the theoretical model can be obtained applying Eq. (29). It should be noted that the accuracy of the temperature measurements
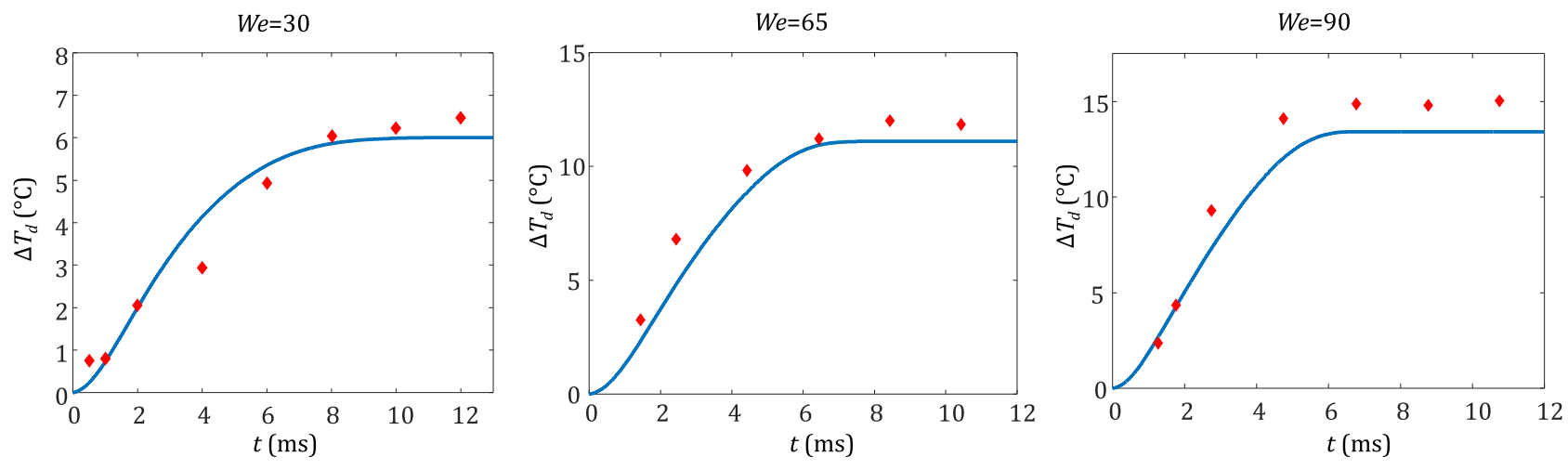

Theoretical model $\quad$ Experiments

FIG. 7. Transient drop heating $\Delta T_{d}$ in the case of ethanol drops impinging onto the sapphire substrate at $T_{w}=300{ }^{\circ} \mathrm{C}\left(T_{d 0}=20{ }^{\circ} \mathrm{C}, d_{0}=1.95 \mathrm{~mm}\right)$. Comparison between the model of drop heating and the measurements of the drop temperature by 2 cLIF thermometry. 
is of the order of $\pm 1{ }^{\circ} \mathrm{C}$. On the other hand, the temperature calculated from the experimental maps is not rigorously the volume-average temperature (see Ref. 28 for an overview of the measurement biases).

In other experiments, the initial drop temperature $T_{d 0}$ was increased from $10{ }^{\circ} \mathrm{C}$ to $70{ }^{\circ} \mathrm{C}$. The experimental results presented in Fig. 8 confirm that $q_{L}$ is proportional to the difference $T_{\text {Sat }}-T_{d 0}$ as predicted by Eqs. (27) and (28). Thus, it can be verified by extrapolating the experimental trends for different Weber numbers that the heat transferred to the drop $q_{L}$ cancels when the initial temperature is close to the saturation.

\section{EVOLUTION OF THE VAPOR FILM THICKNESS}

To determine the evolution of the vapor film, Eq. (20) has to be solved which implies having a description of the reaction force $F$ and of the "contact" diameter $d_{c}$. In Sec. VIII, the resolution is presented using simplified expressions for $F$ and $d_{c}$. In the vast majority of applications, the term in $F$ in Eq. (20) can be neglected as it scales in $h^{3}$. In addition, $F$ decreases exponentially with time when the drop spreads [Eq. (48)]. Replacing $q_{L}$ by its expression in Eq. (27) and assuming $F=0$, the following differential equation can be obtained:

$$
\frac{\mathrm{d} h}{\mathrm{~d} t}=-\frac{\alpha h}{t}+\frac{1}{\rho_{v, s} L v_{e}} \cdot\left(\frac{\lambda_{v} \Delta T}{h}-\frac{C_{q_{L}}}{\sqrt{t}}\right) .
$$

Leaving aside the term $\alpha h / t$, this equation is very similar to that proposed by Breitenbach et al. ${ }^{27}$ to describe the evolution of the vapor film thickness. However, an important difference is that the density of the vapor $\rho_{v, s}$ is replaced by the density of the liquid $\rho_{l}$ [see Eq. (1) in Ref. 27, p. 37], which introduces an overwhelming influence of the evaporation heat flux $q_{v}$ in the energy balance. This difference comes from the fact that Breitenbach et al. ${ }^{27}$ did not consider exactly the same problem. They assume the situation to be the analog of a vapor/liquid front moving in a semi-infinite liquid medium under the effect of phase change without any regard to pressure forces. An analytical solution of Eq. (30) can be found in the form

$$
h(t)=C \sqrt{t} .
$$

Given that $C>0$, the only possible value of $C$ is determined by

$$
C=\frac{-C_{q_{L}}+\sqrt{C_{q_{L}}^{2}+(4 \alpha+2) \rho_{v, s} L v_{e} \lambda_{v} \Delta T}}{\rho_{v, s} L v_{e} \cdot(2 \alpha+1)} .
$$

When $C_{q_{L}}^{2} \gg(4 \alpha+2) \rho_{v, s} L v_{e} \lambda_{v} \Delta T$, a Taylor expansion of Eq. (32) to the 1 st order with respect to $C_{q_{L}}^{-1}$ yields

$$
C=\lambda_{v} \Delta T / C_{q_{L}}
$$

This solution implies that the two predominant terms of Eq. (30) are the heat flux taken to the solid surface $q_{w}=\lambda_{v} \Delta T / h$ and the heat flux entering the liquid phase $q_{L}=C_{q_{L}} / \sqrt{t}$. Indeed, neglecting the other terms, Eq. (30) is equivalent to

$$
\frac{\lambda_{v} \Delta T}{h}=\frac{C_{q_{L}}}{\sqrt{t}}
$$

which immediately yields Eq. (33). The above condition $C_{q_{L}}^{2} \gg(4 \alpha+2) \rho_{v, s} L v_{e} \lambda_{v} \Delta T$ can be rewritten as

$$
\frac{T_{S a t}-T_{d 0}}{T_{w, s}-T_{S a t}} \gg A \cdot \frac{e_{v}}{e_{l}},
$$

where $e_{v}=\sqrt{\rho_{v} C p_{v} \lambda_{v}}$ is the thermal effusivity of vapor and the parameter $A$ is determined by

$$
A=\left[\frac{\pi}{5}(4 \alpha+2) \frac{\rho_{v, s}}{\rho_{v}} \frac{L v_{e}}{C p_{v} \Delta T}\right]^{1 / 2} .
$$

To satisfy the condition in Eq. (35), the initial temperature $T_{d 0}$ must be sufficiently low in comparison to $T_{S a t}$. For example, in the case of an ethanol droplet impinging onto a sapphire substrate at $300{ }^{\circ} \mathrm{C}$, parameter $A$ in Eq. (35) is about equal to 3.4 , while $e_{l} \approx 565 \mathrm{WK}^{-1} \mathrm{~m}^{-2} \mathrm{~s}^{1 / 2}$ and $e_{v} \approx 9.5 \mathrm{WK}^{-1} \mathrm{~m}^{-2} \mathrm{~s}^{1 / 2}$ (evaluated at $T_{\text {film }}$ ). This yields $T_{d 0} \ll 64{ }^{\circ} \mathrm{C}$. Of course, a numerical resolution of the problem will give more accurate estimates of the heat flux transferred to the liquid $q_{L}$ and the heat flux used for evaporation $q_{v a p}$ (see Fig. 14 in Sec. VIII).

\section{SURFACE TEMPERATURE OF THE SOLID WALL}

The cooling of the solid surface can be also taken into account using the same one-dimensional approach ${ }^{27}$ as for the heating of the liquid in Sec. IV. Considering only the thermal gradients in the vertical direction, the problem to address is as follows:

$$
\begin{gathered}
\frac{\partial T}{\partial t}=a_{w} \frac{\partial^{2} T}{\partial z^{2}}, \\
T=T_{w, s} \text { at } z=0, \\
\mathrm{~d} T / \mathrm{d} z=0 \text { at } z=-\infty,
\end{gathered}
$$

where $a_{w}$ is the thermal diffusivity of the solid. The solution of this equation is quite similar to that obtained for Eq. (23)

$$
T_{w}(z, t)=T_{w, s}-\left(T_{w, 0}-T_{w, s}\right) \cdot \operatorname{erf}\left(\frac{z}{2 \sqrt{a_{w} t}}\right),
$$

where $T_{w, 0}$ is the bulk temperature of the sapphire substrate before the drop impact. The heat flux removed from the solid surface can be derived from the following expression:

$$
q_{w}=-\left.\lambda_{w} \frac{\mathrm{d} T}{\mathrm{~d} z}\right|_{z=0^{-}}=\frac{e_{w}\left(T_{w, 0}-T_{w, s}\right)}{\sqrt{\pi t}},
$$

where $e_{w}=\sqrt{\rho_{w} C p_{w} \lambda_{w}}$ is the thermal effusivity of the solid wall. The surface temperature of the wall $T_{w, s}$ can be obtained by comparing the expressions of $q_{w}$ in Eqs. (1) and (41). This yields

$$
q_{w}=\frac{\lambda_{v} \Delta T}{C \sqrt{t}}=\frac{e_{w}\left(T_{w, 0}-T_{w, s}\right)}{\sqrt{\pi t}} .
$$

Remembering that $\Delta T=T_{w, s}-T_{S a t}$ and $C$ is a function of $\Delta T$ according to Eq. (32), Eq. (42) has to be solved to find out the decrease in the temperature of the solid surface, $\Delta T_{w}=T_{w, 0}$ $-T_{w, s}$. The following solution can be obtained:

$$
\frac{1}{8} \frac{\Delta T_{w}=\Delta T_{0}-}{X-\sqrt{64 C_{q_{L}} \sqrt{\pi} e_{w}{ }^{3} \Delta T_{0}-64 \Delta T_{0}^{2} e_{w}^{4}+X^{2}}},
$$


where $X=\pi\left[(4 \alpha+2) \rho_{v, s} L v_{e} \lambda_{v}\right]-4 C_{q_{L}} \sqrt{\pi} e_{w}+8 \Delta T_{0} e_{w}^{2}$ and $\Delta T_{0}=T_{w, 0}-T_{S a t}$. In the case of a cold droplet, i.e., when the condition in Eq. (35) is satisfied (i.e., $q_{L}=q_{w}$ ), $T_{w, s}$ can be simply determined from Eqs. (27) and (41). The decrease in the temperature of the solid surface $\Delta T_{w}$ can be expressed as follows:

$$
\Delta T_{w}=\sqrt{5} \frac{e_{l}}{e_{w}}\left(T_{S a t}-T_{d 0}\right) .
$$

Considering an ethanol droplet, initially at $T_{d 0}=20{ }^{\circ} \mathrm{C}$, impinging onto a sapphire substrate at $T_{w, 0}=300{ }^{\circ} \mathrm{C}\left(e_{w} \approx\right.$ $8300 \mathrm{WK}^{-1} \mathrm{~m}^{-2} \mathrm{~s}^{1 / 2}$ and $e_{l} \approx 565 \mathrm{WK}^{-1} \mathrm{~m}^{-2} \mathrm{~s}^{1 / 2}$ ), Eq. (44) leads to $\Delta T_{w} \approx 9{ }^{\circ} \mathrm{C}$. This value is in good agreement with the measurements of the solid surface temperature presented in Fig. 2(b).

\section{VALIDATION OF THE MODEL IN THE CASE OF $\boldsymbol{F}=0$ AND A LOW INITIAL DROP TEMPERATURE $T_{d 0}$}

As shown in Sec. IV, different cases emerge from the analysis of the evolution of the vapor film thickness. When the liquid drop is sufficiently cold prior to the impact, the condition (35) is satisfied and it is possible to greatly simplify the problem given that $q_{L} \approx q_{w}$. In this section, the focus is on the experimental validation of the model in this limit case. Several experiments were conducted on ethanol droplets injected at the initial temperature of $20{ }^{\circ} \mathrm{C}$. The measurements techniques described in Sec. II are used to determine both the heat transferred to the droplet $Q_{L}$ and the heat taken to the solid surface $Q_{w}$. The heat energy is evaluated from the measurements of the liquid temperature using the following expression:

$$
Q_{L}=m C_{l}\left(T_{m}-T_{d 0}\right),
$$

where $T_{m}$ is the average temperature determined from the measurements using the $2 \mathrm{cLIF}$ technique. The heat $Q_{w}$ is evaluated from the measurements of the solid surface temperature by IR thermography. After determining the heat flux $q_{w}$ by solving the IHCP, $Q_{w}$ is evaluated by

$$
Q_{w}=\int_{0}^{t} \int_{S_{e}(t)} q_{w} \mathrm{~d} S \mathrm{~d} t,
$$

where $S_{e}$ is the cooled surface. In the experiments, the emphasis was placed into the effect of the impact velocity as well as the influence of the surface temperature of the sapphire substrate.

\section{A. Influence of the Weber number}

Figure 9 displays the time evolution of $Q_{L}$ and $Q_{w}$ for different values of the Weber number in the case of an ethanol drop initially at $T_{d 0}=20{ }^{\circ} \mathrm{C}$ and a solid substrate at $T_{w}=$ $300{ }^{\circ} \mathrm{C}$. As expected heat transfer increases with $W e$, which can be explained by the increase in the spreading surface of the droplet. It is noticeable that $Q_{w}$ and $Q_{L}$ are almost equal at any moment of the impact regardless of the Weber number. This confirms that an initial temperature $T_{d 0}=20^{\circ} \mathrm{C}$ is sufficiently low to neglect the contribution of the vaporisation heat flux to the energy balance, as expected from the theory [see the remark at the end of Sec. V after Eq. (36)]. Of course, measurement errors can affect these experimental data, but it
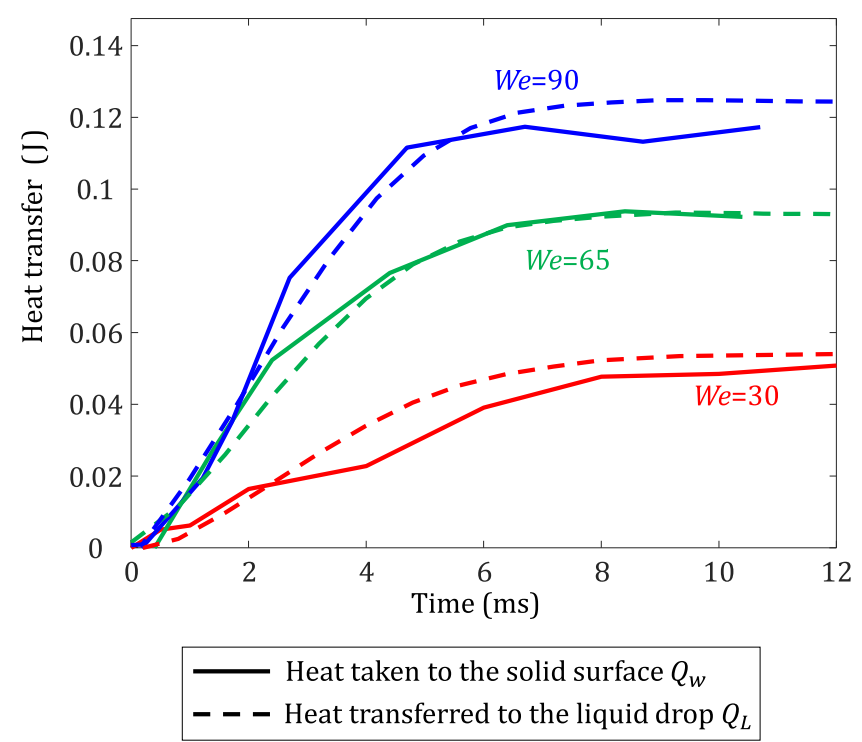

FIG. 9. Time evolution of the heat taken to the solid wall $Q_{W}$ and the heat transferred to the liquid $Q_{L}$ in the case of an ethanol drop $\left(T_{w}=300{ }^{\circ} \mathrm{C}\right.$ and $T_{d 0}=20^{\circ} \mathrm{C}$ ). Experimental data obtained from the measurement of the drop temperature based on laser-induced fluorescence and the measurement of the solid surface temperature using IR thermography.

should be stressed that they are too weak to change the previous conclusion. Indeed, the drop temperature is determined with an uncertainty of about $\pm 1{ }^{\circ} \mathrm{C}$, which corresponds to an energy of $\pm 7.2 \mathrm{~mJ}$. The read noise of the infrared camera is about $\pm 0.2^{\circ} \mathrm{C}$. Given the axisymmetry assumed for solving the IHCP, pixels at the same distance from the center are averaged altogether to reconstruct the local heat flux, which reduces this error. Furthermore, the measurement noise induced by the parasitic radiations from the sapphire holder is drastically reduced, thanks to the deposition of a very emissive coating and the spatial filtering of off-field radiations by the optical system.

Figure 10 shows the time evolution of $h$ for the same impact conditions as in Fig. 9. The thickness of the vapor film

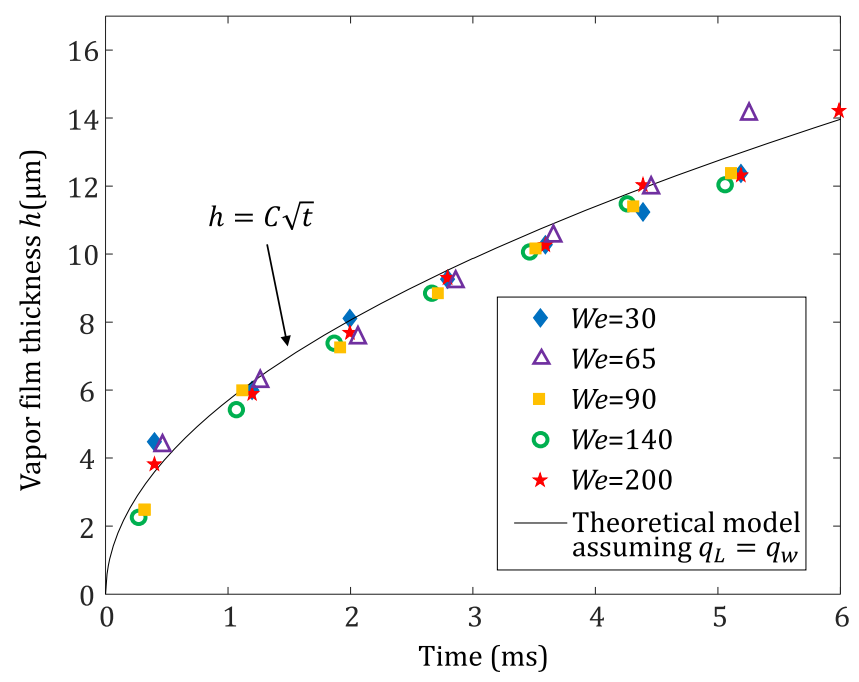

FIG. 10. Time evolution of the thickness $h$ of the vapor film for various Weber numbers in the case of an ethanol droplet impinging the sapphire surface $\left(T_{w}\right.$ $=300{ }^{\circ} \mathrm{C}, T_{d 0}=20^{\circ} \mathrm{C}$ and $\left.d_{0}=1.95 \mathrm{~mm}\right)$. Comparison to the model based on Eqs. (31) and (33). 


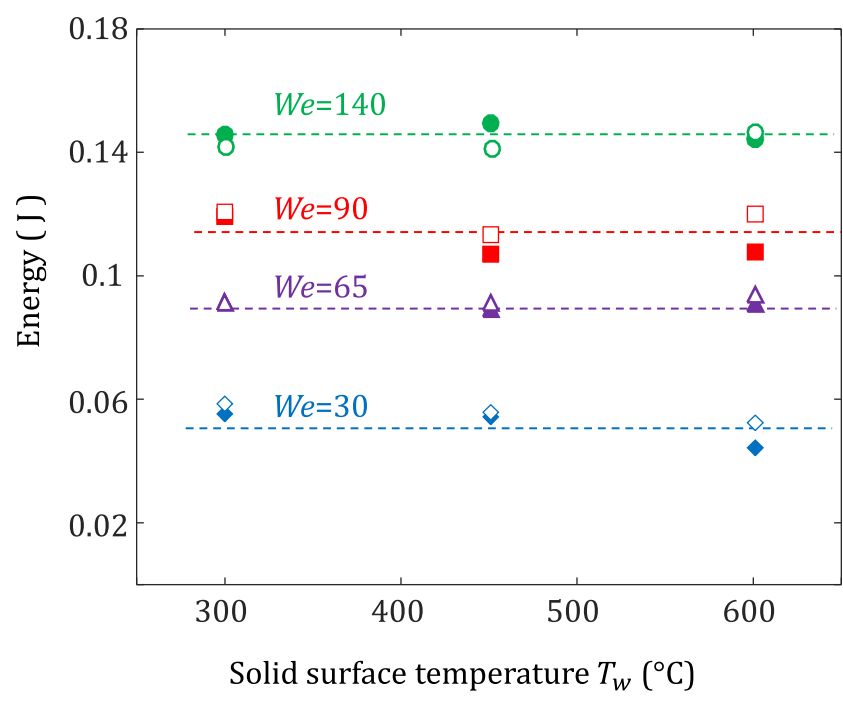

FIG. 11. Influence of the solid surface temperature $T_{w}$ on the heat exchanged between the drop and the solid surface during the drop impact. Hollow symbols correspond to $Q_{L}$ and solid symbols correspond to $Q_{w}$ (ethanol droplet, $T_{d 0}$ $=20^{\circ} \mathrm{C}$ and $d_{0}=1.95 \mathrm{~mm}$ ).

seems to be independent on We. Estimated values of $h$ are only slightly smaller than the prediction of the theoretical model. For these drops impinging the solid surface at $T_{d 0}=20^{\circ} \mathrm{C}$, the evolution of $h$ is well described by Eqs. (31) and (33), which is consistent with the results presented in Fig. 9 suggesting that $q_{w}=q_{L}$.

\section{B. Influence of the solid surface temperature}

The effect of the solid surface temperature is presented in Figs. 11 and 12. The experimental results show that the heat transfer is rather insensitive to the solid surface temperature in the range between $300{ }^{\circ} \mathrm{C}$ and $600{ }^{\circ} \mathrm{C}$. In Fig. 11, the heat exchanged over the complete duration of the impact does not seem to vary significantly with $T_{w}$. Explanations for this behavior can be proposed as follows:

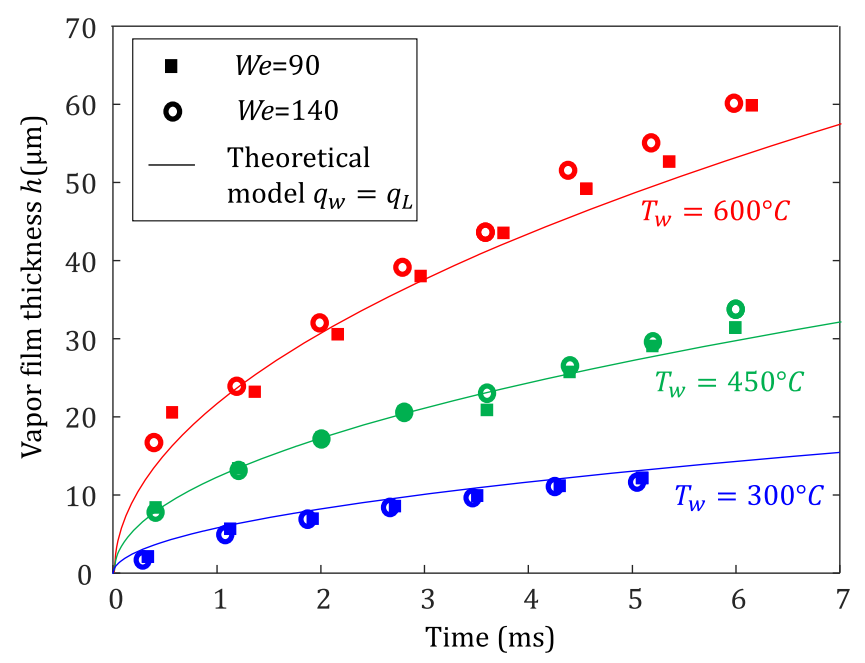

FIG. 12. Time evolution of the vapor film thickness $h$ at different temperatures of the solid surface $T_{w}$ (ethanol droplet, $T_{d 0}=20{ }^{\circ} \mathrm{C}$ and $d_{0}=1.95 \mathrm{~mm}$ ). Comparison to the model based on Eqs. (31) and (33).
- Concerning $Q_{L}$, the drop heating does not depend on the temperature of the solid substrate since the condition $T=T_{\text {Sat }}$ is imposed at the liquid/vapor interface [Eq. (24)]. Furthermore, there is no experimental evidence of a change in the spreading of the drops with the wall temperature in the film boiling regime. ${ }^{27}$

- Concerning $Q_{w}, T_{d 0}=20^{\circ} \mathrm{C}$ is sufficiently low to have still a negligible vaporisation heat flux at $T_{w}=600{ }^{\circ} \mathrm{C}$, even if the limit value for $T_{d 0}$ according to condition (35) decreases with $T_{w}\left(49^{\circ} \mathrm{C}\right.$ at $T_{w}=600{ }^{\circ} \mathrm{C}$ against $64{ }^{\circ} \mathrm{C}$ at $\left.T_{w}=300{ }^{\circ} \mathrm{C}\right)$.

In Fig. 12, the time evolution of the vapor film thickness $h$ is displayed for three different temperatures of the solid surface. Here also, the theoretical model assuming $q_{w}=q_{L}$ is in good agreement with the experimental estimation of the vapor film thickness. Hence, for a sufficiently low initial temperature of the drop, the thickness of the vapor film increases with $T_{w}$ in such a way that $q_{w}$ keeps about the same value.

\section{DISCUSSION IN THE GENERAL CASE $F \neq 0$ AND $T_{d 0} \rightarrow T_{S a t}$}

In the general case, to determine the evolution of the vapor film, it is essential to consider the impact force $F$ and the heat flux devoted to liquid vaporisation $q_{\text {vap }}$. These two contributions tend to play a significant role when the initial drop temperature $T_{d 0}$ comes close to the saturation temperature $T_{\text {Sat }}$. Several studies have proposed expressions for the pressure exerted by the drop on the vapor layer for sessile droplets ${ }^{25}$ or for self-propelled droplets in Leidenfrost state on liquid substrates. ${ }^{20}$ However, little is known about the dynamic force exerted on the vapor film for the impact of a drop. In general, numerical studies dealing with the drop impact in the film boiling regime ${ }^{35,36}$ does not provide enough detailed information to deduce or estimate this force. Roisman et al. ${ }^{6}$ investigated the impact of droplets onto isothermal and perfectly smooth solid surfaces for a wide range of Weber and Reynolds numbers. They found that the pressure under the droplet at $r=0$ can be well approximated by

$$
p_{c} \approx 1.7 \rho_{l} V_{d 0}^{2} \cdot \exp \left(-3.1 t^{*}\right) \text { with } t^{*} \geq 0.2 .
$$

In this expression, $t^{*}=V_{d 0} t / d_{0}$ is the dimensionless impact time. In the following, we assume that Eq. (47) can be applied to the case of a drop in the film boiling regime to evaluate the pressure inside the vapor film. This idea is supported by the fact that the drop deformation (spreading diameter and thickness of the lamella) in the film boiling regime does not differ very much of an impact onto a smooth isothermal surface (at least in the first times when the exerted pressure due to inertial force is still high). Therefore, in the following, $F$ is approximated by

$$
F \approx \frac{\pi d_{c}^{2}}{4} \cdot p_{c}
$$

In order to evaluate $d_{c}$ analytically, a crude approximation is that of a parabolic temporal evolution ${ }^{27}$

$$
d_{c} \approx 4 d_{\max } \cdot\left(\frac{t}{t_{c}}-\frac{t^{2}}{t_{c}^{2}}\right),
$$


a)

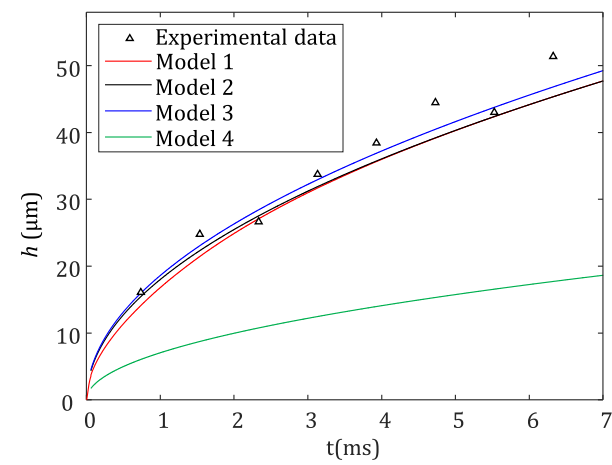

c)

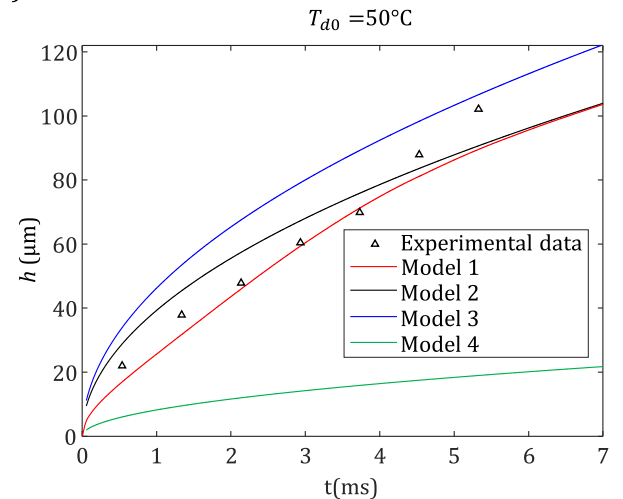

b)

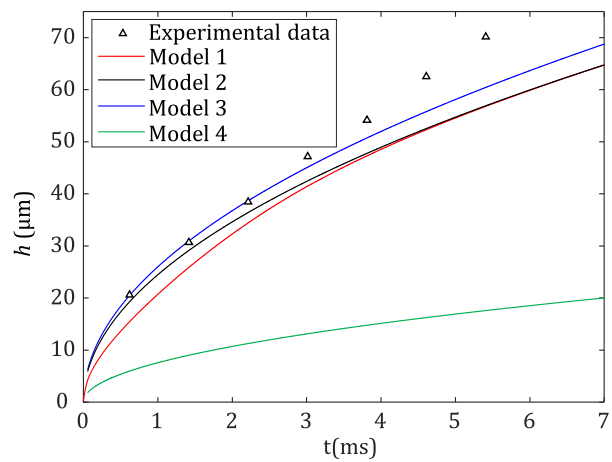

d)

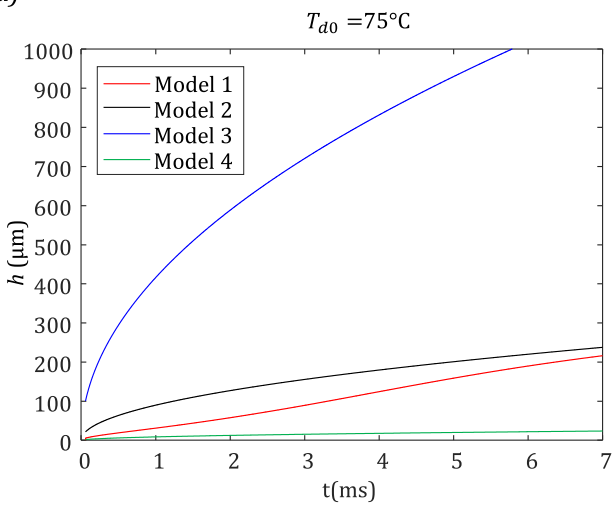

FIG. 13. Time evolution of the vapor film thickness $h$ in the case of an ethanol droplet impinging the sapphire substrate at $T_{w}=600^{\circ} \mathrm{C}$ and $W e=90$. Comparison between the different models mentioned in Sec. VIII and the experiments. where $d_{\max }$ is the maximum spreading diameter. In the case of an ethanol droplet, a value of $d_{\max }$ can be obtained using the following experimental correlation: ${ }^{11}$

$$
d_{\max } / d_{0}=1+0.23 \cdot \sqrt{W e} .
$$

The differential equation [Eq. (20)] describing the dynamical evolution of the vapor film thickness is solved numerically using a variable-step, variable-order method of differentiation given that the problem has an important stiffness. The results presented in Fig. 13 correspond to an ethanol droplet in the impact conditions: $d_{0}=1.95 \mathrm{~mm}, W e=60, T_{w}=600{ }^{\circ} \mathrm{C}$, and $\alpha=1$. The growth of the vapor film can be observed for different initial temperatures of the droplet $T_{d 0}$. Figure 13 allows comparing the different theoretical models described before with the experiments. These models are as follows:

- model 1: the resolution of Eq. (20) with $F$ and $d_{c}$ given by Eqs. (47)-(49),

- model 2: the application of Eq. (32) (assuming that $F$ $=0$ ),

- model 3: the application of Eq. (33) [for a cold droplet satisfying Eq. (35) and $F=0$ ],

- model 4: the theoretical development by Breitenbach et al. ${ }^{27}$

In Fig. 13, all the models listed above predict an increase in the thickness $h$ with the initial drop temperature $T_{d 0}$. However, this increase is much more limited in the case of model 4. The heat used for evaporation has a much larger contribution to the overall balance of heat flux in this model, which assumes $q_{v a p}=L v \rho_{l} \frac{\mathrm{d} h}{\mathrm{~d} t}$. If the comparison is restricted to models $1-3$, the differences between the models are not really significant for moderate initial drop temperatures (in particular, at
$T_{d 0}=11{ }^{\circ} \mathrm{C}$ ), but they drastically increase when $T_{d 0}$ approaches $T_{\text {Sat }}$.

For $T_{d 0} \geq 50{ }^{\circ} \mathrm{C}$, the approximation $q_{w}=q_{L}$ cannot be made any more, since model 3 deviates significantly from models 1 and 2. Model 3 overestimates the growth rate of the vapor film, as it does not account for the heat flux $q_{v a p}$ used for liquid vaporization. However, the contribution of $q_{v a p}$ becomes predominant with the initial drop temperature coming closer to the saturation temperature as illustrated in Figs. 13(d) and 14. The influence of $F$ cannot be ignored in the first $\mathrm{ms}$

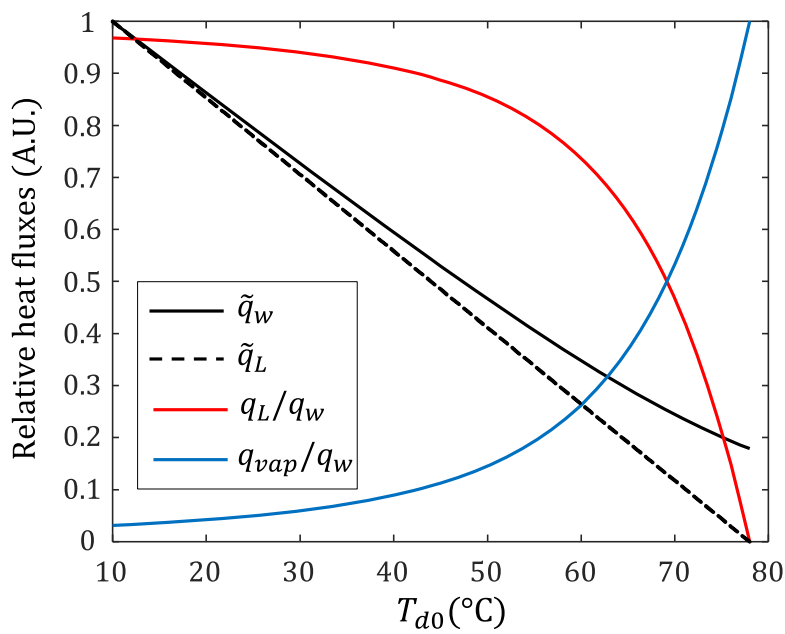

FIG. 14. Evolution of the different heat fluxes involved in the energy balance $q_{w}, q_{L}$, and $q_{v a p}$ with the initial drop temperature $T_{d 0}$ for a solid surface temperature $T_{w}=600^{\circ} \mathrm{C} . \tilde{q}_{w}$ and $\tilde{q}_{L}$ are the variations of $q_{w}$ and $q_{L}$ compared to an impact at $T_{d 0}=10{ }^{\circ} \mathrm{C}$. The results are obtained using model 2 and for the same impact conditions as in Fig. 13. 
of the impact. According to model 1, the pressure induced by the droplet on the vapor film is able to reduce the thickness of the vapor film and modify the balance between the heat fluxes. In response to the pressure exerted by the drop, the film is squeezed which allows enhancing the heat transfer from the wall, more precisely the heat flux due to vaporization $q_{v a p}$ (the evolution of $q_{L}$ being fixed by the condition $T=T_{\text {Sat }}$ at the liquid-vapor interface). This behavior seems to be confirmed by the experiments. It can be seen that the experimental data in Fig. 13(c) are in better agreement with model 1 which takes into account the additional pressure due to the impact. As for the case of $T_{d 0}=30{ }^{\circ} \mathrm{C}$, after $t=5 \mathrm{~ms}$, the experimental data deviate from a $t^{1 / 2}$ evolution (expected theoretically since $F$ tends to 0 with time). The vapor film thickness increases at a faster rate than the models. This deviation is certainly related to the formation of a hole in the liquid lamella as shown in Fig. 2(a) (at $t=6 \mathrm{~ms}$ ). It should be noted that the values of $h$ presented in Fig. 13 correspond to an average over the whole surface of effective heat exchange.

When $T_{d 0}$ is almost equal to $T_{S a t}$, the solution for $h$ diverges toward $+\infty$ for all the models [even model 1 since $p_{c}$ tends rapidly to 0 in Eq. (47)]. It becomes necessary to consider the contribution of the droplet weight, which allows the sessile drop to stabilize over a vapor film of finite thickness as recalled in Eq. (22). Unfortunately, the comparison with the experiments becomes extremely difficult because vapor bubbles are formed at the entrance of the needle of the drop generation system.

\section{CONCLUSIONS}

The evolution of the vapor film thickness has been described in the framework of a quasi-steady approximation, assuming a uniform thickness of the film and one-dimensional heat transfer. A general model for the growth of the vapor film can be obtained from the application of the balance of heat fluxes and the balance of forces applied to the vapor film.

A limit case of the model is obtained when the temperature of the drop before impact is low compared to the saturation temperature of the liquid. In the case of this "cold" drop, heating of the drop outweighs the heat used for the evaporation of the liquid. The growth of the film is governed solely by the rate at which the drop heats up. The impact velocity has no effect on the vapor film. Very simple expressions can be obtained for the evolution of the vapor film thickness and the decrease in temperature of the solid surface. They are validated by experiments conducted on ethanol drops at several wall temperatures and Weber numbers.

Another limit case is that of an initial drop temperature close to the saturation temperature. The pressure exerted by the drop on the vapor film, and thus the impact velocity, has a significant effect on the evolution of the vapor film. The description of this limit case remains very superficial because of a rather rough description of the pressure distribution in the vapor film during its spreading.

\section{ACKNOWLEDGMENTS}

The authors gratefully acknowledge the financial support of the ENERBATIN CPER program and the European FEDER program.

${ }^{1}$ Y. Ji, N. Nagai, and K. Takano, "Effect of inclination angle onto heat transport characteristics of bubble-actuated circulating heat pipe covering high-temperature region," in 15th International Heat Transfer Conference (Begell House Inc. Publishers, 2015), p. 119334.

${ }^{2}$ B. J. Kim and K. D. Kim, "Effect of initial temperature of a cylindrical steel block on heat transfer characteristics of staggered array jets during water jet quenching," Heat Transfer Eng. 36, 1037-1045 (2015).

${ }^{3}$ J. Kim, "Spray cooling heat transfer: The state of the art," Int. J. Heat Fluid Flow 28, 753-767 (2007).

${ }^{4}$ B. Richter, K. Dullenkopf, and H.-J. Bauer, "Investigation of secondary droplet characteristics produced by an isooctane drop chain impact onto a heated piston surface," Exp. Fluids 39, 351-363 (2005).

${ }^{5}$ R. Rioboo, C. Tropea, and M. Marengo, "Outcomes from a drop impact on solid surfaces," Atomization Sprays 11, 155 (2001).

${ }^{6}$ I. V. Roisman, E. Berberović, and C. Tropea, "Inertia dominated drop collisions. I. On the universal flow in the lamella," Phys. Fluids 21, 052103 (2009).

${ }^{7}$ K. Range and F. Feuillebois, "Influence of surface roughness on liquid drop impact," J. Colloid Interface Sci. 203, 16-30 (1998).

${ }^{8}$ X. Li, X. Ma, and Z. Lan, "Behavioral patterns of drop impingement onto rigid substrates with a wide range of wettability and different surface temperatures," AIChE J. 55, 1983-1992 (2009).

${ }^{9}$ G. Liang and I. Mudawar, "Review of drop impact on heated walls," Int. J. Heat Mass Transfer 106, 103-126 (2017).

${ }^{10}$ M. Khavari, C. Sun, D. Lohse, and T. Tran, "Fingering patterns during droplet impact on heated surfaces," Soft Matter 11, 3298-3303 (2015).

${ }^{11}$ G. Castanet, O. Caballina, and F. Lemoine, "Drop spreading at the impact in the Leidenfrost boiling," Phys. Fluids 27, 063302 (2015).

${ }^{12}$ T. Tran, H. J. J. Staat, A. Prosperetti, C. Sun, and D. Lohse, "Drop impact on superheated surfaces," Phys. Rev. Lett. 108, 036101 (2012).

${ }^{13}$ A. Karl and A. Frohn, "Experimental investigation of interaction processes between droplets and hot walls," Phys. Fluids 12, 785-796 (2000).

${ }^{14}$ J.-M. Senoner, G. Castanet, O. Caballina, and P. Villedieu, "Modeling of water drop impactions in the Leidenfrost regime," Atomization Sprays 26, 853-888 (2016)

${ }^{15}$ N. Hatta, H. Fujimoto, K. Kinoshita, and H. Takuda, "Experimental study of deformation mechanism of a water droplet impinging on hot metallic surfaces above the Leidenfrost temperature,” J. Fluids Eng. 119, 692-699 (1997).

${ }^{16}$ A.-L. Biance, F. Chevy, C. Clanet, G. Lagubeau, and D. Quéré, "On the elasticity of an inertial liquid shock," J. Fluid Mech. 554, 47-66 (2006).

${ }^{17}$ A.-L. Biance, C. Pirat, and C. Ybert, "Drop fragmentation due to hole formation during Leidenfrost impact," Phys. Fluids 23, 022104 (2011).

${ }^{18}$ S.-C. Yao and K. Y. Cai, "The dynamics and Leidenfrost temperature of drops impacting on a hot surface at small angles," Exp. Therm. Fluid Sci. 1, 363-371 (1988).

${ }^{19}$ J. Bernardin and I. Mudawar, "A Leidenfrost point model for impinging droplets and sprays," J. Heat Transfer 126, 272-278 (2004).

${ }^{20}$ S. D. Janssens, S. Koizumi, and E. Fried, "Behavior of self-propelled acetone droplets in a Leidenfrost state on liquid substrates," Phys. Fluids 29, 032103 (2017).

${ }^{21}$ J. D. Berry, I. U. Vakarelski, D. Y. C. Chan, and S. T. Thoroddsen, "Navier slip model of drag reduction by Leidenfrost vapor layers," Phys. Fluids 29, 107104 (2017).

${ }^{22}$ B. Sobac, A. Rednikov, S. Dorbolo, and P. Colinet, "Self-propelled Leidenfrost drops on a thermal gradient: A theoretical study," Phys. Fluids 29, 082101 (2017).

${ }^{23}$ G. Dupeux, M. L. Merrer, G. Lagubeau, C. Clanet, S. Hardt, and D. Quéré, "Viscous mechanism for Leidenfrost propulsion on a ratchet," Europhys. Lett. 96, 58001 (2011).

${ }^{24}$ C. Antonini, I. Bernagozzi, S. Jung, D. Poulikakos, and M. Marengo, "Water drops dancing on ice: How sublimation leads to drop rebound," Phys. Rev. Lett. 111, 014501 (2013). 
${ }^{25}$ A.-L. Biance, C. Clanet, and D. Quéré, "Leidenfrost drops," Phys. Fluids 15, 1632-1637 (2003).

${ }^{26}$ B. Sobac, A. Rednikov, S. Dorbolo, and P. Colinet, "Leidenfrost effect: Accurate drop shape modeling and refined scaling laws," Phys. Rev. E 90, 053011 (2014).

${ }^{27}$ J. Breitenbach, I. V. Roisman, and C. Tropea, "Heat transfer in the film boiling regime: Single drop impact and spray cooling," Int. J. Heat Mass Transfer 110, 34-42 (2017).

${ }^{28}$ W. Chaze, O. Caballina, G. Castanet, and F. Lemoine, "Spatially and temporally resolved measurements of the temperature inside droplets impinging on a hot solid surface," Exp. Fluids 58, 96 (2017).

${ }^{29}$ W. Chaze, O. Caballina, G. Castanet, J.-F. Pierson, F. Lemoine, and D. Maillet, "Heat flux reconstruction by inversion of experimental infrared temperature measurements-Application to the impact of a droplet in the film boiling regime," Int. J. Heat Mass Transfer 128, 469-478 (2019).

${ }^{30}$ P. Dunand, G. Castanet, and F. Lemoine, "A two-color planar LIF technique to map the temperature of droplets impinging onto a heated wall," Exp. Fluids 52, 843-856 (2012).
${ }^{31}$ G. Castanet, T. Lienart, and F. Lemoine, "Dynamics and temperature of droplets impacting onto a heated wall," Int. J. Heat Mass Transfer 52, 670-679 (2009).

${ }^{32}$ Y. Guo and K. Mishima, "A non-equilibrium mechanistic heat transfer model for post-dryout dispersed flow regime," Exp. Therm. Fluid Sci. 26, 861-869 (2002).

${ }^{33}$ A. L. Yarin and D. A. Weiss, "Impact of drops on solid surfaces: Self-similar capillary waves, and splashing as a new type of kinematic discontinuity," J. Fluid Mech. 283, 141-173 (1995).

${ }^{34}$ J. Stefan, "Versuch über die scheinbere adhäsion," Sitzungsberichte der Mathematisch-naturwissenschaften Klasse der Kaiserlichen Akademie der Wissenschaften, II. Abteilung, 1874, pp. 713-735.

${ }^{35}$ Y. Ge and L.-S. Fan, "Three-dimensional simulation of impingement of a liquid droplet on a flat surface in the Leidenfrost regime," Phys. Fluids 17, 027104 (2005).

${ }^{36}$ L. R. Villegas, S. Tanguy, G. Castanet, O. Caballina, and F. Lemoine, "Direct numerical simulation of the impact of a droplet onto a hot surface above the Leidenfrost temperature," Int. J. Heat Mass Transfer 104, 1090-1109 (2017). 